\title{
Finite-Time Stabilization for Discrete-Time Delayed Markovian Jump Systems with Partially Delayed Actuator Saturation
}

\author{
Guoliang Wang and Bo Feng \\ School of Information and Control Engineering, Liaoning Shihua University, Fushun, Liaoning 113001, China \\ Correspondence should be addressed to Guoliang Wang; gliangwang@aliyun.com
}

Received 19 August 2016; Revised 23 October 2016; Accepted 25 October 2016

Academic Editor: Zhengqiu Zhang

Copyright ( 2016 G. Wang and B. Feng. This is an open access article distributed under the Creative Commons Attribution License, which permits unrestricted use, distribution, and reproduction in any medium, provided the original work is properly cited.

\begin{abstract}
The finite-time control problem of discrete-time delayed Markovian jump systems with partially delayed actuator saturation is considered by a mode-dependent parameter approach. Different from the traditionally saturated actuators, a kind of saturated actuator being partially delay-dependent is firstly proposed, where both nondelay and delay states are included and occur asynchronously. Moreover, the probability distributions of such two terms are described by the Bernoulli variable and are taken into account in the controller design. Sufficient conditions for the existence of the desired controller are presented with LMIs. Finally, a numerical example is provided to show the effectiveness and superiority of the obtained results.
\end{abstract}

\section{Introduction}

As we know, Markovian jump systems (MJSs) are very appropriate to describe dynamical systems experiencing changes in their structures or parameters randomly. Up to now, many important research topics of this kind of system, such as stability analysis [1-4], stabilization [5-7], $H_{\infty}$ control and filtering [8-11], output control [12, 13], state estimation [14, $15]$, adaptive control $[16,17]$, and synchronization $[18,19]$, have been considered. By investigating most results on the stability in the literature, it is seen that the classical Lyapunov stability guaranteeing the stability in an infinite-time interval was usually considered. Different from the classical Lyapunov stability concept, finite-time stability is defined as the system state that does not exceed a certain bound during a fixed finite-time interval. The introduction of such a stability concept is very necessary and important in many practical applications, where the related quantities need to fall into a range with specified bounds in a fixed-time interval. Up to now, many results, such as finite-time stability [20-23], stabilization [24, 25], finite-time $H_{\infty}$ control or filtering [2628], and input-output finite-time stability and stabilization $[29,30]$, have emerged.

On the other hand, many dynamical systems experience physical constraints, where actuator saturation is a common control problem because the amplitude of the actuator signal is limited by physical, technological, or even safety constraints. It is very known that actuator saturation could lead to poor performance of the closed-loop systems and even instability. Because of its practical and theoretical importance, stability analysis and synthesis for control systems with actuator or sensor saturation have received a lot of attention [31-36]. When the considered system is an MJS, some results have been proposed such as [37-41]. By investigating the above results on actuator saturation, it is found that they are mainly based on an estimate of the domain of attraction, where an equivalent description of saturation actuator is needed. However, the introduction of this transformation will make the complexity of computation very large, especially for an MJS with $\mathrm{N}$ operation modes. By introducing an inequality to enlarge some terms related to saturation [42], some delay-dependent stabilization results with less complexity were obtained in [43]. It is seen that the introduced inequality is mainly used in the derivative of Lyapunov function. Very recently, another different inequality was introduced in [44], which was based on an equivalent definition and was firstly used in the system description. By investigating such references, it is found that the designed state feedback controllers including delayed systems are only related to nondelay state. This means that the corresponding state should be available online. It will be impossible in some practical applications, such as systems 
communicating through an unreliable network, where the transmitted information is not obtained on time but has time delay sometimes. In other words, the event of data transmitted with or without time delay happens randomly. Moreover, it is known that one cannot say that either of the controllers only with nondelay or delay states is less conservative or not. It is natural and better to consider them together and design a kind of controller where both nondelay and delay states are contained but occur asynchronously. To the best of our knowledge, very few results are available to study the above problems. All the observations motivate the current research.

In this paper, we address the finite-time stabilization for a class of discrete-time delayed Markovian jump systems, where the actuator saturation is partially delay-dependent. The main contributions of this paper are summarized as follows. (1) A new kind of saturated controller being partially delay-dependent is proposed, where both nondelay and delay states are included but happen randomly. Moreover, the related probability distribution is embodied by the Bernoulli variable and is taken into account in its design. (2) Sufficient conditions checking the finite-time stability of the underlying system are presented with LMI forms, where a modedependent parameter approach is exploited successfully. Particularly, some techniques in terms of additional inequalities and variables are proposed to obtain the LMI conditions ultimately. (3) Compared with the similar results about finitetime stabilization of MJSs with input saturation, the proposed methods have some advantages in terms of being solved easily and being less conservative. (4) The effects of time delay and actuator saturation are fully considered, which are important in system analysis and synthesis. Moreover, because of the results within LMI framework, they could be extended to other general cases directly.

Notation. $\mathbb{R}^{n}$ denotes the $n$-dimensional Euclidean space, and $\mathbb{R}^{m \times n}$ is the set of all $m \times n$ real matrices. $\mathscr{E}\{\cdot\}$ means the mathematical expectation of $\{\cdot\} .\|\cdot\|$ refers to the Euclidean vector norm or spectral matrix norm. $\mathbb{Z}^{+}$is the set of positive integers. $\Omega$ is the sample space, $\mathscr{F}$ is the $\sigma$-algebras of subsets of the sample space, and $\mathbb{P}$ is the probability measure on $\mathscr{F}$. In symmetric block matrices, we use "*” as an ellipsis for the terms induced by symmetry, $\operatorname{diag}\{\cdots\}$ for a block-diagonal matrix, and $(M)^{\star} \triangleq M+M^{T}$.

\section{Problem Formulation}

Consider the following discrete-time delayed Markovian jump system (DDMJS) subject to actuator saturation:

$$
\begin{aligned}
x(k+1)= & A\left(r_{k}\right) x(k)+A_{d}\left(r_{k}\right) x(k-d) \\
& +B\left(r_{k}\right) \text { sat }(u(k)), \\
x(k)= & \phi(k),
\end{aligned}
$$

where $x(k) \in \mathbb{R}^{n}$ is the state vector, $u(k) \in \mathbb{R}^{q}$ is the control input, and $\phi(k) \in \mathbb{R}^{n}$ is the initial condition. Matrices $A\left(r_{k}\right), A_{d}\left(r_{k}\right)$, and $B\left(r_{k}\right)$ are known matrices of compatible dimensions. $\left\{r_{k}, k \in \mathbb{Z}^{+}\right\}$is a discrete-time Markov process taking values in a finite space $\mathbb{S}=\{1,2, \ldots, N\}$ with TRM $\Pi \triangleq\left(\pi_{i j}\right) \in \mathbb{R}^{N \times N}$ given by

$$
\pi_{i j}=\operatorname{Pr}\left\{r_{k+1}=j \mid r_{k}=i\right\},
$$

where $0 \leq \pi_{i j} \leq 1$ and $\sum_{j=1}^{N} \pi_{i j}=1$ for all $i \in \mathbb{S}$. Time delay $d$ satisfies $d>0$. Function $\operatorname{sat}(\cdot): \mathbb{R}^{q} \rightarrow \mathbb{R}^{q}$ is the standard function defined as

$$
\operatorname{sat}\left(u_{i}(k)\right)=\left[\operatorname{sat}\left(u_{1}(k)\right) \cdots \operatorname{sat}\left(u_{q}(k)\right)\right]^{T},
$$

where $\operatorname{sat}\left(u_{i}(k)\right)=\operatorname{sign}\left(u_{i}(k)\right) \min \left\{1,\left|u_{i}(k)\right|\right\}$.

In this paper, the designed state feedback controller is named to be a partially delay-dependent controller and is described by

$$
\begin{aligned}
u(k)= & \alpha(k) K\left(r_{k}\right) x(k) \\
& +(1-\alpha(k)) K_{d}\left(r_{k}\right) x(k-d),
\end{aligned}
$$

where $K\left(r_{k}\right)$ and $K_{d}\left(r_{k}\right)$ are the control gains to be determined. Stochastic variable $\alpha(k)$ is an indicator and is defined as follows:

$$
\alpha(k)= \begin{cases}1, & \text { if } x(k) \text { is available } \\ 0, & \text { if } x(k-d) \text { is available }\end{cases}
$$

which is satisfied as follows:

$$
\begin{aligned}
& \operatorname{Pr}\{\alpha(k)=1\}=\mathscr{E}\{\alpha(k)\}=\alpha, \\
& \operatorname{Pr}\{\alpha(k)=0\}=1-\alpha .
\end{aligned}
$$

Then, the resulting closed-loop system is rewritten to be

$$
\begin{aligned}
& x(k+1)=A\left(r_{k}\right) x(k)+A_{d}\left(r_{k}\right) x(k-d)+B\left(r_{k}\right) \\
& \quad \cdot \operatorname{sat}\left(\alpha(k) K\left(r_{k}\right) x(k)\right. \\
& \left.\quad+(1-\alpha(k)) K_{d}\left(r_{k}\right) x(k-d)\right), \\
& x(k)=\phi(k) .
\end{aligned}
$$

Definition 1. Given scalars $c_{2}>0, D>0$ and matrix $R_{i}>0$, DDMJS (1) with $u(k)=0$ is said to be stochastic finite-time stable with respect to $\Gamma_{i}=\left(c_{1}, c_{2}, D, R_{i}\right)$, if there is a positive real scalar $c_{1}$, possibly depending on $c_{2}, D$, and $R_{i}$, such that

$$
\begin{aligned}
& \mathscr{E}\left\{x^{T}\left(k_{1}\right) R_{i} x\left(k_{1}\right)\right\} \leq c_{1} \Longrightarrow \\
& \mathscr{E}\left\{x^{T}\left(k_{2}\right) R_{i} x\left(k_{2}\right)\right\} \leq c_{2},
\end{aligned}
$$

where $k_{1} \in\{-d,-d+1, \ldots,-1,0\}$ and $k_{2} \in\{1,2, \ldots, D\}$.

By letting $\eta=u-\operatorname{sat}(u)$, it is known that

$$
\begin{aligned}
\operatorname{sat}(u(k))= & \alpha(k) K\left(r_{k}\right) x(k) \\
& +(1-\alpha(k)) K_{d}\left(r_{k}\right) x(k-d) \\
& -\left[\alpha(k) \eta\left(r_{k}\right)+(1-\alpha(k)) \eta_{d}\left(r_{k}\right)\right]
\end{aligned}
$$


which is equivalent to

$$
\begin{aligned}
& \text { sat }(u(k))=\alpha K\left(r_{k}\right) x(k)+(1-\alpha) K_{d}\left(r_{k}\right) x(k-d) \\
& \quad+(\alpha(k)-\alpha)\left(K\left(r_{k}\right) x(k)-K_{d}\left(r_{k}\right) x(k-d)\right) \\
& \quad-\left[\alpha \eta\left(r_{k}\right)+(1-\alpha) \eta_{d}\left(r_{k}\right)\right. \\
& \left.\quad+(\alpha(k)-\alpha)\left(\eta\left(r_{k}\right)-\eta_{d}\left(r_{k}\right)\right)\right]
\end{aligned}
$$

Then, the resulting closed-loop system becomes

$$
\begin{aligned}
& x(k+1)=A\left(r_{k}\right) x(k)+A_{d}\left(r_{k}\right) x(k-d)+B\left(r_{k}\right) \\
& \cdot\left(\alpha K\left(r_{k}\right) x(k)+(1-\alpha) K_{d}\left(r_{k}\right) x(k-d)+(\alpha(k)\right. \\
& -\alpha)\left(K\left(r_{k}\right) x(k)-K_{d}\left(r_{k}\right) x(k-d)\right)-\left(\alpha \eta\left(r_{k}\right)\right. \\
& \left.\left.\quad+(1-\alpha) \eta_{d}\left(r_{k}\right)+(\alpha(k)-\alpha)\left(\eta\left(r_{k}\right)-\eta_{d}\left(r_{k}\right)\right)\right)\right), \\
& x(k)=\phi(k) .
\end{aligned}
$$

Remark 2. It is said that actuator saturation with description (4) is more general and has some advantages. Firstly, compared with input saturation $[31,36,37,39,41,44]$ where nondelay state should be available online, system state $x(k)$ in description (4) is not necessary and could be replaced by delay state $x(k-d)$. Secondly, compared with designed saturated controllers $[35,43]$ which were designed for delayed systems without delay states, both nondelay and delay states are included in the designed saturated controller. As we know, because all the delay terms affect the results, it is more conservative without considering them. Then, it is said that our formulation about the actuator saturation will have a larger application scope and less conservatism, since both related states are involved, and theirs distribution probabilities are also considered.

Lemma 3 (see [44]). Let $\eta=u-\operatorname{sat}(u)$. Then, there exists $a$ real number $\epsilon \in(0,1)$ such that

$$
\eta^{T} \eta \leq \epsilon u^{T} u
$$

where $\eta=\left[\begin{array}{lll}\eta_{1} & \cdots & \eta_{q}\end{array}\right]^{T}$ and $\eta_{i}$ is the dead-zone nonlinearity function, $i=\{1,2, \ldots, q\}$.

\section{Main Results}

Theorem 4. Given a saturation controller (9), the resulting closed-loop system (11) is finite-time stable with respect to $\Gamma_{i}$, if, for given positive scalars $\mu_{i} \in[1, \infty), \epsilon_{i} \in(0,1)$, and $\epsilon_{d i} \in(0,1)$, there exist $P_{i}>0, Q>0, \gamma_{i}>0, \delta_{1}>0, \delta_{2}>0$, and $v_{2}>0$, such that

$$
\left[\begin{array}{cccccc}
\Theta_{i 1} & 0 & \Theta_{i 2} & \Theta_{i 3} & \Theta_{i 4} & 0 \\
* & -Q & \Theta_{i 5} & \Theta_{i 6} & 0 & \Theta_{i 7} \\
* & * & -\bar{P}_{i} & 0 & 0 & 0 \\
* & * & * & -\bar{P}_{i} & 0 & 0 \\
* & * & * & * & -\gamma_{i} I & 0 \\
* & * & * & * & * & -\gamma_{i} I
\end{array}\right]<0,
$$

$\left[\begin{array}{cc}-\gamma_{i} I & B_{i}^{T} \bar{P}_{i} \\ * & -\bar{P}_{i}\end{array}\right] \leq 0$

$$
\begin{aligned}
\delta_{1} I<\widehat{P}_{i}<\delta_{2} I, \\
0<\widehat{Q}_{i}<v_{2} I,
\end{aligned}
$$

$$
\mu_{i}^{D}\left(\delta_{2}+d v_{2}\right) c_{1} \leq \delta_{1} c_{2}
$$

where

$$
\begin{aligned}
& \Theta_{i 1}=Q-\mu_{i} P_{i}, \\
& \Theta_{i 2}=\sqrt{2} \bar{A}_{i}^{T} \bar{P}_{i}, \\
& \bar{A}_{i}=A_{i}+\alpha B_{i} K_{i}, \\
& \bar{P}_{i}=\sum_{j=1}^{N} \pi_{i j} P_{j} \\
& \Theta_{i 3}=\sqrt{2 \alpha(1-\alpha)} K_{i}^{T} B_{i}^{T} \bar{P}_{i}, \\
& \Theta_{i 4}=\sqrt{\left(6 \alpha-4 \alpha^{2}\right) \epsilon_{i}} \gamma_{i} K_{i}^{T} \\
& \Theta_{i 5}=\sqrt{2} \bar{A}_{d i}^{T} \bar{P}_{i}, \\
& \bar{A}_{d i}=A_{d i}+(1-\alpha) B_{i} K_{d i}, \\
& \Theta_{i 6}=-\sqrt{2 \alpha(1-\alpha)} K_{d i}^{T} B_{i}^{T} \bar{P}_{i} \\
& \Theta_{i 7}=\sqrt{\left(2 \alpha-4 \alpha^{2}+2\right) \epsilon_{d i}} \gamma_{i} K_{d i}^{T} \text {, } \\
& \widehat{P}_{i}=R_{i}^{-1 / 2} P_{i} R_{i}^{-1 / 2}, \\
& \widehat{Q}_{i}=R_{i}^{-1 / 2} Q R_{i}^{-1 / 2} \text {. }
\end{aligned}
$$

Proof. Choose a stochastic Lyapunov function for system (11) as

$$
V(k)=x^{T}(k) P\left(r_{k}\right) x(k)+\sum_{l=k-d}^{k-1} x^{T}(l) Q x(l) .
$$


Then, it is further obtained that

$$
\begin{aligned}
\mathscr{E}\{ & V(k+1)\}=\left(\bar{A}_{i} x(k)+\bar{A}_{d i} x(k-d)\right)^{T} \\
& \bar{P}_{i}\left(\bar{A}_{i} x(k)+\bar{A}_{d i} x(k-d)\right)+\alpha(1-\alpha) \\
\cdot & \left(K_{i} x(k)-K_{d i} x(k-d)-\left(\eta_{i}-\eta_{d i}\right)\right)^{T} \\
\cdot & B_{i}^{T} \bar{P}_{i} B_{i}\left(K_{i} x(k)-K_{d i} x(k-d)-\left(\eta_{i}-\eta_{d i}\right)\right) \\
& +\left[\alpha \eta_{i}+(1-\alpha) \eta_{d i}\right]^{T} B_{i}^{T} \bar{P}_{i} B_{i}\left[\alpha \eta_{i}+(1-\alpha) \eta_{d i}\right] \\
& -2\left(\bar{A}_{i} x(k)+\bar{A}_{d i} x(k-d)\right)^{T} \\
& \bar{P}_{i} B_{i}\left[\alpha \eta_{i}+(1-\alpha) \eta_{d i}\right]+\sum_{l=k+1-d}^{k} x^{T}(l) Q x(l) \\
& =f_{i 1}^{T} \bar{P}_{i} f_{i 1}+\alpha(1-\alpha) f_{i 2}^{T} B_{i}^{T} \bar{P}_{i} B_{i} f_{i 2} \\
& +f_{i 3}^{T} B_{i}^{T} \bar{P}_{i} B_{i} f_{i 3}-2 f_{i 1}^{T} \bar{P}_{i} B_{i} f_{i 3}+\sum_{l=k+1-d}^{k} x^{T}(l) Q x(l) \\
& \leq 2 f_{i 1}^{T} \bar{P}_{i} f_{i 1}+\alpha(1-\alpha) f_{i 2}^{T} B_{i}^{T} \bar{P}_{i} B_{i} f_{i 2} \\
& +2 f_{i 3}^{T} B_{i}^{T} \bar{P}_{i} B_{i} f_{i 3}+\sum_{l=k+1-d}^{k} x^{T}(l) Q x(l)
\end{aligned}
$$

where

$$
\begin{aligned}
& f_{i 1}=\bar{A}_{i} x(k)+\bar{A}_{d i} x(k-d), \\
& f_{i 2}=f_{i 4}-\left(\eta_{i}-\eta_{d i}\right), \\
& f_{i 3}=\alpha \eta_{i}+(1-\alpha) \eta_{d i}, \\
& f_{i 4}=K_{i} x(k)-K_{d i} x(k-d) .
\end{aligned}
$$

Moreover, it is concluded that

$$
\begin{gathered}
\alpha(1-\alpha) f_{i 2}^{T} B_{i}^{T} \bar{P}_{i} B_{i} f_{i 2}=\alpha(1-\alpha)\left[f_{i 4}-\left(\eta_{i}-\eta_{d i}\right)\right]^{T} \\
\cdot B_{i}^{T} \bar{P}_{i} B_{i}\left[f_{i 4}-\left(\eta_{i}-\eta_{d i}\right)\right] \leq 2 \alpha(1-\alpha) \\
\cdot\left[f_{i 4}^{T} B_{i}^{T} \bar{P}_{i} B_{i} f_{i 4}+\left(\eta_{i}-\eta_{d i}\right)^{T} B_{i}^{T} \bar{P}_{i} B_{i}\left(\eta_{i}-\eta_{d i}\right)\right] \\
\leq 2 \alpha(1-\alpha) f_{i 4}^{T} B_{i}^{T} \bar{P}_{i} B_{i} f_{i 4}+4 \alpha(1-\alpha) \\
\cdot\left(\eta_{i}^{T} B_{i}^{T} \bar{P}_{i} B_{i} \eta_{i}+\eta_{d i}^{T} B_{i}^{T} \bar{P}_{i} B_{i} \eta_{d i}\right), \\
2 f_{i 3}^{T} B_{i}^{T} \bar{P}_{i} B_{i} f_{i 3}=2\left[\alpha \eta_{i}+(1-\alpha) \eta_{d i}\right]^{T} \\
\cdot B_{i}^{T} \bar{P}_{i} B_{i}\left[\alpha \eta_{i}+(1-\alpha) \eta_{d i}\right] \\
\leq 2\left[\alpha \eta_{i}^{T} B_{i}^{T} \bar{P}_{i} B_{i} \eta_{i}+(1-\alpha) \eta_{d i}^{T} B_{i}^{T} \bar{P}_{i} B_{i} \eta_{d i}\right] .
\end{gathered}
$$

Based on this, it is computed that

$$
\begin{aligned}
\Delta V(k)= & \mathscr{E}\left\{V r_{k}(k+1)=, i r_{k+1}=j\right\}-V(k) \\
\leq & 2 f_{i 1}^{T} \bar{P}_{i} f_{i 1}+2 \alpha(1-\alpha) f_{i 4}^{T} B_{i}^{T} \bar{P}_{i} B_{i} f_{i 4} \\
& +\left(6 \alpha-4 \alpha^{2}\right) \eta_{i}^{T} B_{i}^{T} \bar{P}_{i} B_{i} \eta_{i}
\end{aligned}
$$

$$
\begin{aligned}
& +\left(2 \alpha-4 \alpha^{2}+2\right) \eta_{d i}^{T} B_{i}^{T} \bar{P}_{i} B_{i} \eta_{d i} \\
& +\sum_{l=k+1-d}^{k} x^{T}(l) Q x(l)-x^{T}(k) P_{i} x(k) \\
& -\sum_{l=k-d}^{k-1} x^{T}(l) Q x(l) .
\end{aligned}
$$

From condition (14), it is seen that

$$
B_{i}^{T} \bar{P}_{i} B_{i} \leq \gamma_{i} I .
$$

By Lemma 3, we have

$$
\begin{aligned}
\alpha(k) & \eta_{i}^{T} \eta_{i}+(1-\alpha(k)) \eta_{d i}^{T} \eta_{d i} \\
\leq & \epsilon_{i} \alpha(k)\left(K_{i} x(k)\right)^{T} K_{i} x(k) \\
& \quad+\epsilon_{d i}(1-\alpha(k))\left(K_{d i} x(k-d)\right)^{T} K_{d i} x(k-d) .
\end{aligned}
$$

Based on these, it is got that (23) is implied by

$$
\begin{aligned}
& \Delta V(k) \leq 2\left(\bar{A}_{i} x(k)+\bar{A}_{d i} x(k-d)\right)^{T} \bar{P}_{i}\left(\bar{A}_{i} x(k)\right. \\
& \left.+\bar{A}_{d i} x(k-d)\right)+2 \alpha(1-\alpha)\left(K_{i} x(k)-K_{d i} x(k-d)\right)^{T} \\
& \text { - } B_{i}^{T} \bar{P}_{i} B_{i}\left(K_{i} x(k)-K_{d i} x(k-d)\right)+\left(6 \alpha-4 \alpha^{2}\right) \gamma_{i} \epsilon_{i} x^{T}(k) \\
& \cdot K_{i}^{T} K_{i} x(k)+\left(2 \alpha-4 \alpha^{2}+2\right) \gamma_{i} \epsilon_{d i} x^{T}(k-d) K_{d i}^{T} K_{d i} x(k \\
& -d)+\sum_{l=k+1-d}^{k} x^{T}(l) Q x(l)-x^{T}(k) P_{i} x(k) \\
& -\sum_{l=k-d}^{k-1} x^{T}(l) Q x(l)=\xi^{T}(K)\left(\left[\begin{array}{cc}
Q-P_{i} & 0 \\
0 & -Q
\end{array}\right]\right. \\
& +2\left[\begin{array}{c}
\bar{A}_{i}^{T} \\
\bar{A}_{d i}^{T}
\end{array}\right] \bar{P}_{i}\left[\begin{array}{c}
\bar{A}_{i}^{T} \\
\bar{A}_{d i}^{T}
\end{array}\right]^{T} \\
& +2 \alpha(1-\alpha)\left[\begin{array}{c}
K_{i}^{T} B_{i}^{T} \\
-K_{d i}^{T} B_{i}^{T}
\end{array}\right] \bar{P}_{i}\left[\begin{array}{c}
K_{i}^{T} B_{i}^{T} \\
-K_{d i}^{T} B_{i}^{T}
\end{array}\right]^{T} \\
& \left.+\left[\begin{array}{cc}
\left(6 \alpha-4 \alpha^{2}\right) \epsilon_{i} \gamma_{i} K_{i}^{T} K_{i} & 0 \\
0 & \left(2 \alpha-4 \alpha^{2}+2\right) \epsilon_{d i} \gamma_{i} K_{d i}^{T} K_{d i}
\end{array}\right]\right) \\
& \cdot \xi(k)=\xi^{T}(K)\left(\left[\begin{array}{cc}
Q-\mu_{i} P_{i} & 0 \\
0 & -Q
\end{array}\right]+2\left[\begin{array}{c}
\bar{A}_{i}^{T} \\
\bar{A}_{d i}^{T}
\end{array}\right] \bar{P}_{i}\left[\begin{array}{c}
\bar{A}_{i}^{T} \\
\bar{A}_{d i}^{T}
\end{array}\right]^{T}\right. \\
& +2 \alpha(1-\alpha)\left[\begin{array}{c}
K_{i}^{T} B_{i}^{T} \\
-K_{d i}^{T} B_{i}^{T}
\end{array}\right] \bar{P}_{i}\left[\begin{array}{c}
K_{i}^{T} B_{i}^{T} \\
-K_{d i}^{T} B_{i}^{T}
\end{array}\right]^{T} \\
& \left.+\left[\begin{array}{cc}
\left(6 \alpha-4 \alpha^{2}\right) \epsilon_{i} \gamma_{i} K_{i}^{T} K_{i} & 0 \\
0 & \left(2 \alpha-4 \alpha^{2}+2\right) \epsilon_{d i} \gamma_{i} K_{d i}^{T} K_{d i}
\end{array}\right]\right) \\
& \cdot \xi(k)+\left(\mu_{i}-1\right) x^{T}(t) P_{i} x(t),
\end{aligned}
$$


where

$$
\xi^{T}(k)=\left[\begin{array}{lll}
x^{T}(k) & x^{T}(k-d)
\end{array}\right] .
$$

After applying the Schur complementary lemma to (13), it is concluded that (26) implies

$$
\begin{aligned}
\Delta V(k) & =\mathscr{E}\{V(k+1)\}-V(k) \\
& \leq\left(\mu_{i}-1\right) x^{T}(k) P_{i} x(k) \leq\left(\mu_{i}-1\right) V(k) .
\end{aligned}
$$

Therefore, one has

$$
\mathscr{E}\{V(k+1)\} \leq \mu_{i} V(k) .
$$

It follows that

$$
\mathscr{E}\{V(k)\} \leq \mu_{i}^{k} \mathscr{E}\{V(0)\} .
$$

Based on the definitions of $\widehat{P}_{i}$ and $\widehat{Q}_{i}$, it is obtained that

$$
\begin{aligned}
\mathscr{E}\{V(0)\}= & \mathscr{E}\left\{x^{T}(0) R_{i}^{1 / 2} \widehat{P}_{i} R_{i}^{1 / 2} x(0)\right\} \\
& +\mathscr{E}\left\{\sum_{-d}^{-1} x^{T}(l) R_{i}^{1 / 2} \widehat{Q}_{i} R_{i}^{1 / 2} x(l)\right\} \\
\leq & c_{1} \sup _{i \in \mathbb{S}} \lambda_{\max }\left(\widehat{P}_{i}\right)+d c_{1} \sup _{i \in \mathbb{S}} \lambda_{\max }\left(\widehat{Q}_{i}\right),
\end{aligned}
$$

where $d \in \mathbb{Z}^{+}$. On the other hand, it is known from (19) that

$$
\begin{aligned}
\mathscr{E}\{V(k)\} & \geq \mathscr{E}\left\{x^{T}(k) P_{i} x(k)\right\} \\
& =\mathscr{E}\left\{x^{T}(k) R_{i}^{1 / 2} \widehat{P}_{i} R_{i}^{1 / 2} x(k)\right\} \\
& \geq \inf _{i \in \mathbb{S}} \lambda_{\min }\left(\widehat{P}_{i}\right) \mathscr{E}\left\{x^{T}(k) R_{i} x(k)\right\} .
\end{aligned}
$$

Taking into account (30)-(32), we obtain that

$$
\begin{aligned}
\inf _{i \in \mathbb{S}} \lambda_{\min }\left(\widehat{P}_{i}\right) \mathscr{E}\left\{x^{T}(k) R_{i} x(k)\right\} \\
\quad \leq \mu_{i}^{k} c_{1}\left(\sup _{i \in \mathbb{S}} \lambda_{\max }\left(\widehat{P}_{i}\right)+d \sup _{i \in \mathbb{S}} \lambda_{\max }\left(\widehat{Q}_{i}\right)\right) .
\end{aligned}
$$

Based on conditions (15)-(17), it is obtained that

$$
\mathscr{E}\left\{x^{T}(k) R_{i} x(k)\right\} \leq \frac{\mu_{i}^{D}\left[\delta_{2}+d v_{2}\right] c_{1}}{\delta_{1}} \leq c_{2},
$$

where $k \in\{1,2, \ldots, D\}$. This completes the proof.

Remark 5. In this paper, it is seen that a mode-dependent parameter approach in terms of $\Delta V(k) \leq\left(\mu_{i}-1\right) V(k)$ is exploited to deal with the finite-time stability problem of DDMJS (1) with actuator saturation (9). Instead of choosing a common scalar $\mu$ for all system modes, the modedependent parameter $\mu_{i}$ is selected. Similar to the results in [23], it is known that the corresponding results will be less conservative. Moreover, in order to obtain LMI conditions, some necessarily additional variables and inequalities are introduced to be computed, which are not given beforehand.
Remark 6. From the proof of this theorem, it is seen that the obtained result is based on the constructed Lyapunov functional (19). It is said the selected Lyapunov functional is without loss of generality. This means that it could be replaced by an improved or another one, where more information of time delay or some double summable quadratic terms could be used in the construction of Lyapunov functional. It is common that less conservative results could be obtained, when more information about time delay is used. Moreover, in order to further reduce the conservatism, some improved techniques referred to as slack variable method and Jensen inequality approach may be applied here too. However, because of the so many terms coming from a complex Lyapunov functional in addition to exploiting improved techniques, the detailed steps should be revisited carefully. In other words, they cannot be applied directly or easily to study the proposed problems. For example, when a more complex Lyapunov functional and improved techniques are applied, more terms and variables will be introduced, which will make the obtained results have large computation complexity. Moreover, some additional problems will emerge. How to get the LMI conditions is one of them. In a word, all the factors will make the problems very complicated, which will be considered in our future work.

Theorem 7. Given a scalar $\alpha \in[0,1]$, there exists a saturation controller (9) such that the resulting system is finite-time stable with respect to $\Gamma_{i}$, if, for given positive scalars $\mu_{i} \in[1, \infty), \epsilon_{i} \in$ $(0,1)$, and $\epsilon_{d i} \in(0,1)$, there exist $X_{i}>0, \bar{Q}>0, \bar{\gamma}_{i}>0, \delta_{1}>0$, $\delta_{2}>0, v_{2}>0, K_{i}$ and $K_{d i}$ satisfying conditions (17) and

$$
\begin{aligned}
& {\left[\begin{array}{ccccccc}
-\mu_{i} X_{i} & 0 & \Psi_{i 1} & \Psi_{i 2} & \Psi_{i 3} & 0 & X_{i} \\
* & -2 X_{i}+\bar{Q} & \Psi_{i 4} & \Psi_{i 5} & 0 & \Psi_{i 6} & 0 \\
* & * & -\bar{X} & 0 & 0 & 0 & 0 \\
* & * & * & -\bar{X} & 0 & 0 & 0 \\
* & * & * & * & -\bar{\gamma}_{i} I & 0 & 0 \\
* & * & * & * & * & -\bar{\gamma}_{i} I & 0 \\
* & * & * & * & * & * & -\bar{Q}
\end{array}\right]<0,} \\
& {\left[\begin{array}{cc}
-\bar{\gamma}_{i} I & \Phi \\
* & -\bar{X}
\end{array}\right] \leq 0,} \\
& \delta_{1} I-2 R_{i}^{-1 / 2}+X_{i}<0, \\
& {\left[\begin{array}{cc}
-\delta_{2} I & R_{i}^{-1 / 2} \\
* & -X_{i}
\end{array}\right]<0,} \\
& {\left[\begin{array}{cc}
-v_{2} I & R_{i}^{-1 / 2} \\
* & -\bar{Q}
\end{array}\right]<0,}
\end{aligned}
$$




$$
\begin{aligned}
& \Psi_{i 1}=\left[\sqrt{2 \pi_{i 1}} X_{i} A_{i}^{T}+\alpha \sqrt{2 \pi_{i 1}} Y_{i}^{T} B_{i}^{T} \cdots \sqrt{2 \pi_{i N}} X_{i} A_{i}^{T}+\alpha \sqrt{2 \pi_{i N}} Y_{i}^{T} B_{i}^{T}\right],
\end{aligned}
$$

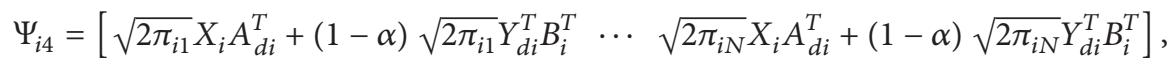

$$
\begin{aligned}
& \Psi_{i 2}=\left[\begin{array}{lll}
\sqrt{\left(2 \alpha-2 \alpha^{2}\right) \pi_{i 1}} Y_{i}^{T} B_{i}^{T} & \cdots \sqrt{\left(2 \alpha-2 \alpha^{2}\right) \pi_{i N}} Y_{i}^{T} B_{i}^{T}
\end{array}\right], \\
& \Psi_{i 5}=\left[-\sqrt{\left(2 \alpha-2 \alpha^{2}\right) \pi_{i 1}} Y_{d i}^{T} B_{i}^{T} \cdots-\sqrt{\left(2 \alpha-2 \alpha^{2}\right) \pi_{i N}} Y_{d i}^{T} B_{i}^{T}\right], \\
& \Psi_{i 3}=\sqrt{\left(6 \alpha-4 \alpha^{2}\right) \epsilon_{i}} Y_{i}^{T} \text {, } \\
& \Psi_{i 6}=\sqrt{\left(2 \alpha-4 \alpha^{2}+2\right) \epsilon_{d i}} Y_{d i}^{T} \text {, } \\
& \Phi=\left[\begin{array}{lll}
\sqrt{\pi_{i 1}} \bar{\gamma}_{i} B_{i}^{T} & \cdots & \sqrt{\pi_{i N}} \bar{\gamma}_{i} B_{i}^{T}
\end{array}\right] \\
& \bar{X}=\operatorname{diag}\left\{X_{1}, \ldots, X_{N}\right\} \text {. }
\end{aligned}
$$

Then, the gains of saturation controller (9) are computed by

$$
\begin{gathered}
K_{i}=Y_{i} X_{i}^{-1}, \\
K_{d i}=Y_{d i} X_{i}^{-1} .
\end{gathered}
$$

Proof. From condition (26), it is known that

$$
\begin{aligned}
& \Delta V(k) \leq \xi^{T}(k)\left(\left[\begin{array}{cc}
Q-\mu_{i} P_{i} & 0 \\
0 & -Q
\end{array}\right]\right. \\
& +\sqrt{2}\left[\begin{array}{c}
\bar{A}_{i}^{T} \\
\bar{A}_{d i}^{T}
\end{array}\right] \bar{P}_{i} \sqrt{2}\left[\begin{array}{c}
\bar{A}_{i}^{T} \\
\bar{A}_{d i}^{T}
\end{array}\right]^{T} \\
& +\sqrt{2 \alpha(1-\alpha)}\left[\begin{array}{c}
K_{i}^{T} B_{i}^{T} \\
-K_{d i}^{T} B_{i}^{T}
\end{array}\right] \bar{P}_{i} \sqrt{2 \alpha(1-\alpha)}\left[\begin{array}{c}
K_{i}^{T} B_{i}^{T} \\
-K_{d i}^{T} B_{i}^{T}
\end{array}\right]^{T} \\
& \left.+\left[\begin{array}{cc}
\left(6 \alpha-4 \alpha^{2}\right) \epsilon_{i} \gamma_{i} K_{i}^{T} K_{i} & 0 \\
0 & \left(2 \alpha-4 \alpha^{2}+2\right) \epsilon_{d i} \gamma_{i} K_{d i}^{T} K_{d i}
\end{array}\right]\right) \\
& \cdot \xi(k)+\left(\mu_{i}-1\right) x^{T}(t) P_{i} x(t) .
\end{aligned}
$$

By the Schur complement lemma, it is obtained that

$$
\left[\begin{array}{cccccc}
\bar{\Psi}_{i 1} & 0 & \bar{\Psi}_{i 2} & \bar{\Psi}_{i 3} & \bar{\Psi}_{i 4} & 0 \\
* & -Q & \bar{\Psi}_{i 5} & \bar{\Psi}_{i 6} & 0 & \bar{\Psi}_{i 7} \\
* & * & -\bar{X} & 0 & 0 & 0 \\
* & * & * & -\bar{X} & 0 & 0 \\
* & * & * & * & -\bar{\gamma}_{i} I & 0 \\
* & * & * & * & * & -\bar{\gamma}_{i} I
\end{array}\right]<0
$$

where

$$
\begin{aligned}
& \bar{\Psi}_{i 1}=Q-\mu_{i} X_{i}^{-1}, \\
& X_{i}=P_{i}^{-1} \text {, } \\
& \bar{\Psi}_{i 2}=\left[\begin{array}{lll}
\sqrt{2 \pi_{i 1}} \bar{A}_{i}^{T} & \cdots & \sqrt{2 \pi_{i N}} \bar{A}_{i}^{T}
\end{array}\right], \\
& \bar{\Psi}_{i 5}=\left[\begin{array}{lll}
\sqrt{2 \pi_{i 1}} \bar{A}_{d i}^{T} & \cdots & \sqrt{2 \pi_{i N}} \bar{A}_{d i}^{T}
\end{array}\right], \\
& \bar{\Psi}_{i 3}=\left[\begin{array}{lll}
\sqrt{\left(2 \alpha-2 \alpha^{2}\right) \pi_{i 1}} K_{i}^{T} B_{i}^{T} & \cdots & \sqrt{\left(2 \alpha-2 \alpha^{2}\right) \pi_{i N}} K_{i}^{T} B_{i}^{T}
\end{array}\right], \\
& \bar{\Psi}_{i 6} \\
& =\left[-\sqrt{\left(2 \alpha-2 \alpha^{2}\right) \pi_{i 1}} K_{d i}^{T} B_{i}^{T} \cdots-\sqrt{\left(2 \alpha-2 \alpha^{2}\right) \pi_{i N}} K_{d i}^{T} B_{i}^{T}\right], \\
& \bar{\Psi}_{i 4}=\sqrt{\left(6 \alpha-4 \alpha^{2}\right) \epsilon_{i}} K_{i}^{T}, \\
& \bar{\Psi}_{i 7}=\sqrt{\left(2 \alpha-4 \alpha^{2}+2\right) \epsilon_{d i}} K_{d i}^{T}, \\
& \bar{\gamma}_{i}=\gamma_{i}^{-1} \text {. }
\end{aligned}
$$

By pre- and postmultiplying its both sides with $\operatorname{diag}\left\{X_{i}, X_{i}, I\right.$, $I, I, I\}$ and its transpose and taking into account (41), one gets

$$
\left[\begin{array}{cccccc}
\Omega_{i 1} & 0 & \Psi_{i 1} & \Psi_{i 2} & \Psi_{i 3} & 0 \\
* & \Omega_{i 2} & \Psi_{i 4} & \Psi_{i 5} & 0 & \Psi_{i 6} \\
* & * & -\bar{X} & 0 & 0 & 0 \\
* & * & * & -\bar{X} & 0 & 0 \\
* & * & * & * & -\bar{\gamma}_{i} I & 0 \\
* & * & * & * & * & -\bar{\gamma}_{i} I
\end{array}\right]<0,
$$

where

$$
\begin{aligned}
& \Omega_{i 1}=X_{i} Q X_{i}-\mu_{i} X_{i}, \\
& \Omega_{i 2}=-X_{i} Q X_{i} .
\end{aligned}
$$

As for $\Omega_{i 2}$, it is obvious that $\Omega_{i 2}<0$ can be guaranteed by

$$
-2 X_{i}+\bar{Q}<0,
$$


where $\bar{Q}=Q^{-1}$. By applying the Schur complement lemma again and considering condition (47), it is concluded that condition (35) implies (45). As for condition (24), it is equivalent to

$$
-\bar{\gamma}_{i} I+\bar{\gamma}_{i}^{2} B_{i}^{T} \sum_{j=1}^{N} \pi_{i j} P_{j} B_{i} \leq 0
$$

which is equal to (36). As for condition (15), it is equivalent to

$$
\begin{gathered}
\delta_{1} I-R_{i}^{-1 / 2} P_{i} R_{i}^{-1 / 2}<0, \\
-\delta_{2} I+R_{i}^{-1 / 2} P_{i} R_{i}^{-1 / 2}<0,
\end{gathered}
$$

which could be implied by (37) and (38), respectively. Similarly, it is known that (16) will be guaranteed by (39). This completes the proof.

Remark 8 . Because of probability $\alpha$ included obviously, it is said that it is important to the analysis and synthesis of systems with actuator saturation. When $\alpha=a$, saturated controller (9) will be simplified to one in [44]. On the contrary, one will get a saturated controller only related to delay state. Based on these explanations, it is said that our results can be viewed as extension results on the saturation stabilization problem from deterministic systems without time delay to stochastic delay systems. However, it is seen that such a probability should be given exactly. It will be impossible or will be of high cost in some practical applications. Instead, we only have its estimation. In this case, there will be an uncertainty between the real and estimated values. As we know, such an uncertainty will degrade the system performance and even lead to system instability. Thus, it is necessary and meaningful to consider this general case. Because of the obtained results with LMI forms here, it is concluded that they could be extended to the above general case, where some existing methods such as $[5,10]$ will be used to deal with this uncertainty.

Remark 9. It is worth mentioning that the inequality in Lemma 3 plays an essential role in dealing with the saturated terms introduced by the new partially delay-dependent controller. By investigating such an inequality, it is actually a nonlinear function satisfying a global Lipschitz condition. Recently, some new LMI-based sufficient conditions were proposed in $[45,46]$, which were obtained by applying LMI method and homeomorphism theory simultaneously. Moreover, it has been proved in these references that the extended global Lipschitz condition on the activation functions is less conservative than the traditionally global Lipschitz conditions. By considering the methods proposed in this paper and such two references together, some new LMI conditions may be proposed, which will have less conservatism. However, how to obtain the LMI conditions ultimately should be considered carefully. Many steps should be revisited, where some new problems may be encountered. All the related problems will be considered in our future work.

\section{Numerical Examples}

In this section, a numerical example is used to show the applicability and superiority of the proposed methods.

Example 1. Consider a discrete-time delayed Markovian jump system of form (1) having two modes, whose parameters are described as follows:

Mode 1:

$$
\begin{aligned}
A_{1} & =\left[\begin{array}{cc}
-0.6 & 0.3 \\
0.2 & 0.1
\end{array}\right], \\
A_{d 1} & =\left[\begin{array}{cc}
0.2 & 0 \\
0.1 & 0.1
\end{array}\right], \\
B_{1} & =\left[\begin{array}{l}
-1 \\
0.5
\end{array}\right],
\end{aligned}
$$

Mode 2:

$$
\begin{aligned}
A_{2} & =\left[\begin{array}{cc}
0.2 & 0.4 \\
0.1 & -0.3
\end{array}\right], \\
A_{d 2} & =\left[\begin{array}{cc}
0.2 & -0.1 \\
0.3 & -0.1
\end{array}\right], \\
B_{2} & =\left[\begin{array}{c}
0.2 \\
1
\end{array}\right],
\end{aligned}
$$

where matrices $R_{1}>0$ and $R_{2}>0$ are given to be

$$
\begin{aligned}
& R_{1}=\left[\begin{array}{ll}
1 & 0 \\
0 & 1
\end{array}\right], \\
& R_{2}=\left[\begin{array}{ll}
1 & 0 \\
0 & 1
\end{array}\right] .
\end{aligned}
$$

The transition rate matrix is given by

$$
\Pi=\left[\begin{array}{ll}
0.8 & 0.2 \\
0.3 & 0.7
\end{array}\right] .
$$

Without loss of generality, letting $T=2, c_{1}=1, c_{2}=8, d=3$, $D=4, \mu_{1}=1.3, \mu_{2}=1.2, \epsilon_{1}=0.4, \epsilon_{2}=0.5, \epsilon_{d 1}=0.3$, and $\epsilon_{d 2}=0.4$, by Theorem 7 , we have the gains of saturated controller (9) with $\alpha=0.6$ computed as

$$
\begin{aligned}
K_{1} & =\left[\begin{array}{ll}
-0.0298 & 0.0146
\end{array}\right], \\
K_{d 1} & =\left[\begin{array}{ll}
0.0214 & -0.0013
\end{array}\right], \\
K_{2} & =\left[\begin{array}{ll}
-0.0230 & -0.0026
\end{array}\right], \\
K_{d 2} & =\left[\begin{array}{ll}
-0.0135 & 0.0031
\end{array}\right] .
\end{aligned}
$$

Under the initial condition $x_{0}=\left[\begin{array}{ll}0.2 & -0.2\end{array}\right]$, after applying the above saturated controller, one has the simulation of the resulting closed-loop system given in Figure 1, where 


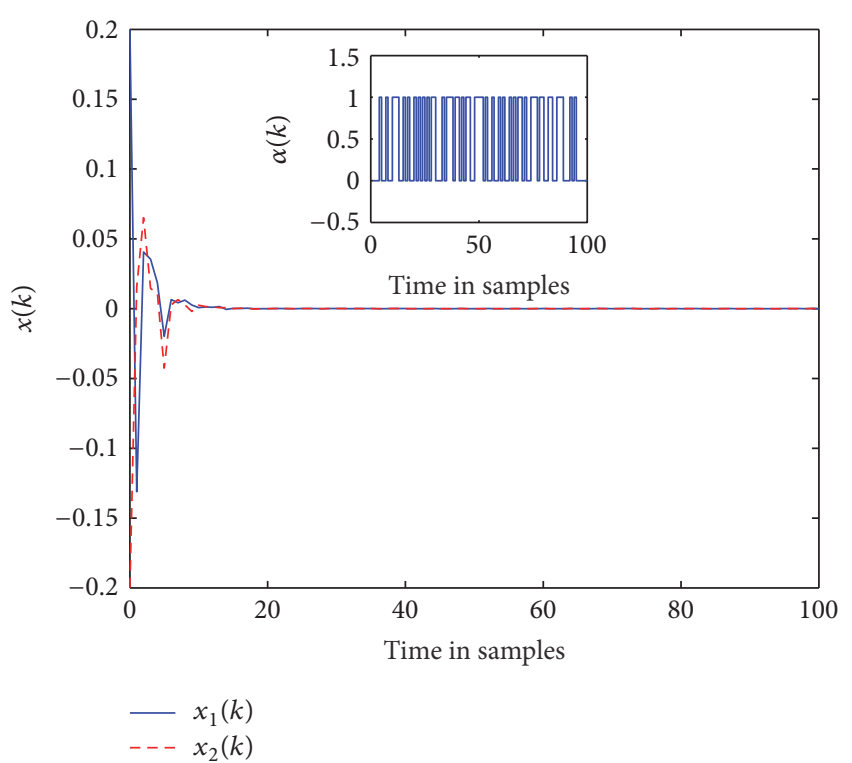

Figure 1: The simulation of the resulting closed-loop system.

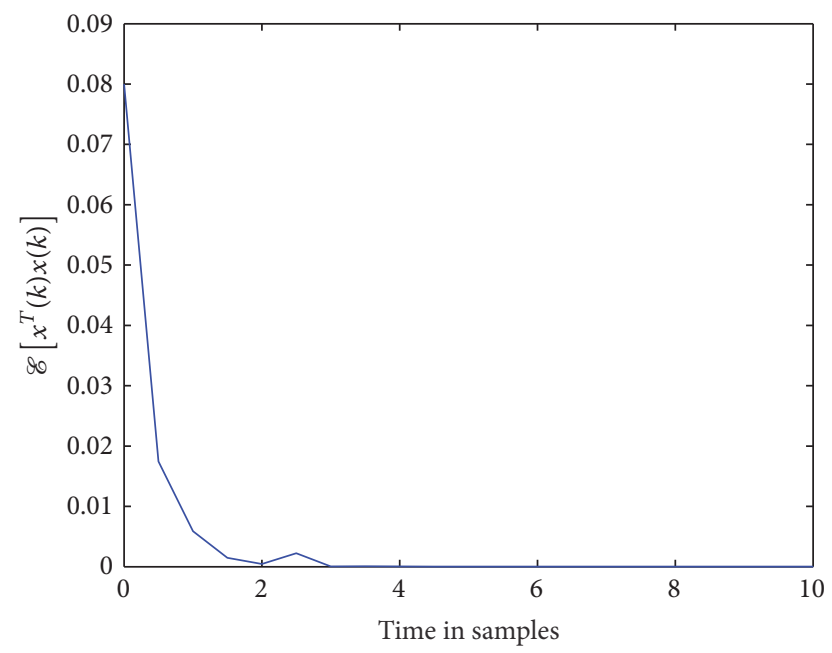

Figure 2: The curve of $\mathscr{E}\left\{x^{T}(k) x(k)\right\}$.

the smaller figure is the simulation of stochastic variable $\alpha(k)$ with $\alpha=0.6$. Moreover, the curve of $\mathscr{E}\left\{x^{T}(k) x(k)\right\}$ is simulated in Figure 2. Based on these simulations, it is said that the designed saturated controller with form (9) could make the resulting system stochastic finite-time stable with respect to $\Gamma_{i}=\left(c_{1}, c_{2}, D, R_{i}\right)$, which also demonstrates the utility of the proposed methods.

In order to further demonstrate the efficiency of the proposed methods and the correlation among such given parameters, without loss of generality, the above matrix $A_{1}$ is replaced by

$$
A_{1}=\left[\begin{array}{cc}
-0.3+\delta & 0.3 \\
0.2 & 0.1
\end{array}\right] \text {, }
$$

TABLE 1: The allowable minimum $\alpha$ for different pair $\left(\mu_{1}, \mu_{2}\right)$.

\begin{tabular}{lcccc}
\hline & $\mu_{2}=1.0$ & $\mu_{2}=1.1$ & $\mu_{2}=1.2$ & $\mu_{2}=1.28$ \\
\hline$\mu_{1}=1.0$ & 0.84 & 0.83 & 0.88 & 0.98 \\
$\mu_{1}=1.1$ & 0.71 & 0.70 & 0.74 & 0.81 \\
$\mu_{1}=1.2$ & 0.60 & 0.60 & 0.60 & 0.67 \\
$\mu_{1}=1.28$ & 0.55 & 0.54 & 0.54 & 0.55 \\
\hline
\end{tabular}

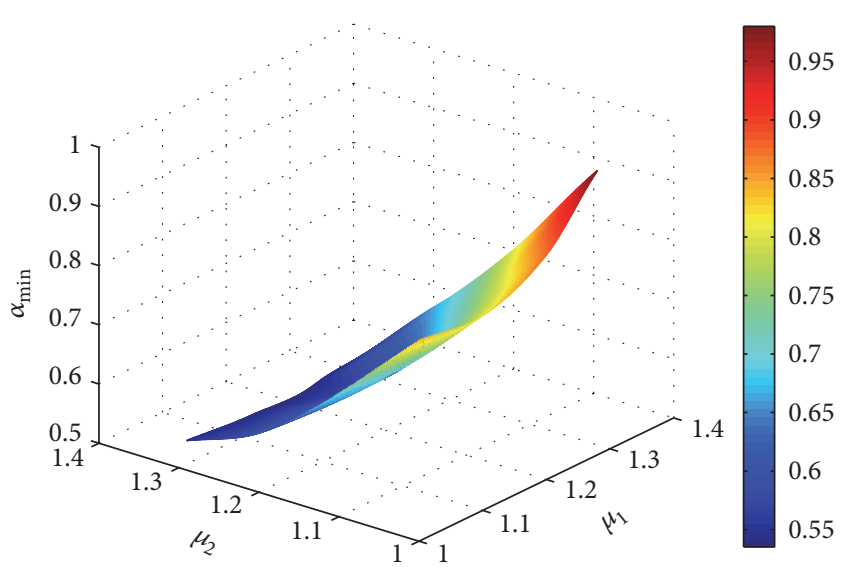

FIGURE 3: The simulation of correlation between pair $\left(\mu_{1}, \mu_{2}\right)$ and $\alpha_{\min }$.

where $\delta$ is a scalar, and the other matrices are invariant. Firstly, we demonstrate the correlation between modedependent parameter $\mu_{i}$ and expectation $\alpha$. Without loss of generality, by letting $\delta=-0.359$ and from Theorem 7 , one gets the allowable minimum $\alpha$ such that the resulting system could be stochastic finite-time stable by the saturated controller (9) under different mode-dependent pair $\left(\mu_{1}, \mu_{2}\right)$, given in Table 1 . By denoting the allowable minimum $\alpha$ as $\alpha_{\min }$ and based on Table 1 , we have the simulation of correlation between pair $\left(\mu_{1}, \mu_{2}\right)$ and $\alpha_{\text {min }}$ given in Figure 3. In order to further demonstrate the correlation between pair $\left(\mu_{1}, \mu_{2}\right)$ and $\alpha$, the allowable maximum of $\mu_{2}$ along with $\mu_{1}$ under different given $\alpha$ is given in Table 2 , where ""denotes the notion that there is no solution to $\mu_{2}$. Then, the corresponding simulation is presented in Figure 4. As for this case, it is concluded that larger probability $\alpha$ will lead to less conservative results. This means that, for a given $\mu_{1} \geq 1$, the range of the allowable maximum of $\mu_{2}$ with larger $\alpha$ will be larger. Moreover, it is found that the difference of such an allowable range along with $\mu_{1}$ will be smaller even when $\alpha$ has different values. This phenomenon could be used to design a better saturated controller (9). Based on these simulations, it is found that mode-dependent parameters are important in system synthesis, which could make the results less conservative in terms of smaller $\alpha_{\min }$.

Secondly, we illustrate the effect of expectation $\alpha$ on finite-time stabilization by a partially delay-dependent controller with saturation (3). Under the selected parameters same as the above ones, the allowable maximum and minimum of $\delta$ denoted as $\delta_{\max }$ and $\delta_{\min }$, respectively, could be obtained from Theorem 7 , which are given in Table 3. Based on this table, we could get the curves of parameters $\delta_{\max }$, 
TABLE 2: The allowable maximum $\mu_{2}$ along with $\mu_{1}$ for different $\alpha$.

\begin{tabular}{cccccc}
\hline & $\alpha=0.6$ & $\alpha=0.7$ & $\alpha=0.8$ & $\alpha=0.9$ & $\alpha=1$ \\
\hline$\mu_{1}=1.00$ & - & - & - & 1.22 & 1.29 \\
$\mu_{1}=1.03$ & - & - & 1.13 & 1.27 & 1.30 \\
$\mu_{1}=1.06$ & - & - & 1.21 & 1.29 & 1.30 \\
$\mu_{1}=1.10$ & - & 1.15 & 1.29 & 1.30 & 1.30 \\
$\mu_{1}=1.15$ & - & 1.24 & 1.30 & 1.30 & 1.30 \\
$\mu_{1}=1.20$ & 1.20 & 1.30 & 1.29 & 1.30 & 1.30 \\
$\mu_{1}=1.28$ & 1.30 & 1.30 & 1.29 & 1.30 & 1.30 \\
\hline
\end{tabular}

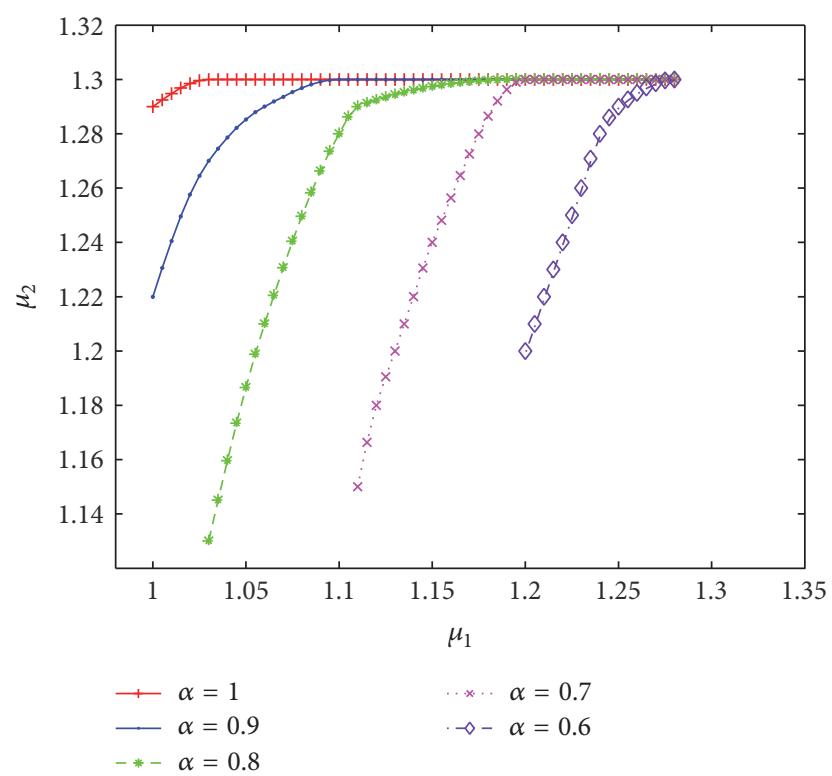

FIgURE 4: The curves of $\mu_{2}$ along with $\mu_{1}$ under different $\alpha$.

TABLE 3: The allowable maximum and minimum $\delta$ for different $\alpha$.

\begin{tabular}{lccccc}
\hline$\alpha$ & 0 & 0.3 & 0.5 & 0.8 & 1 \\
\hline$\delta_{\max }$ & 0.779 & 0.769 & 0.758 & 0.768 & 0.798 \\
$\delta_{\min }$ & -0.245 & -0.298 & -0.350 & -0.470 & -0.565 \\
\hline
\end{tabular}

$\delta_{\min }$, and $\delta_{\text {ran }} \triangleq \delta_{\max }-\delta_{\text {min }}$ along with $\alpha$ simulated in Figure 5. From this simulation, it is seen that probability $\alpha$ has a positive effect on this case in terms of $\delta_{\text {ran }}$ having a larger value.

Now, we consider the effects of parameters $\epsilon_{i}$ and $\epsilon_{d i}$ on $\delta_{\text {ran }}$. First of all, the value of $\delta_{\text {ran }}$ along with different pair $\left(\epsilon_{1}, \epsilon_{2}\right)$ is studied. Based on Theorem 7 where the other parameters are invariant, $\delta_{\text {ran }}$ under different pair $\left(\epsilon_{1}, \epsilon_{2}\right)$ could be got, listed in Table 4 . Then, the correlation between pair $\left(\epsilon_{1}, \epsilon_{2}\right)$ and $\delta_{\text {ran }}$ is simulated in Figure 6. Similarly, the corresponding results and simulations about pair $\left(\epsilon_{d 1}, \epsilon_{d 2}\right)$ and $\delta_{\text {ran }}$ are presented in Table 5 and Figure 7 . Based on these simulations, it is said that different values of such parameters have different effects and could make the results have different conservatism in terms of $\delta_{\text {ran }}$ being larger or not. For this case, it is seen that smaller values of $\left(\epsilon_{1}, \epsilon_{2}\right)$ and $\left(\epsilon_{d 1}, \epsilon_{d 2}\right)$ will lead to less conservative results in terms of larger $\delta_{\text {ran }}$.
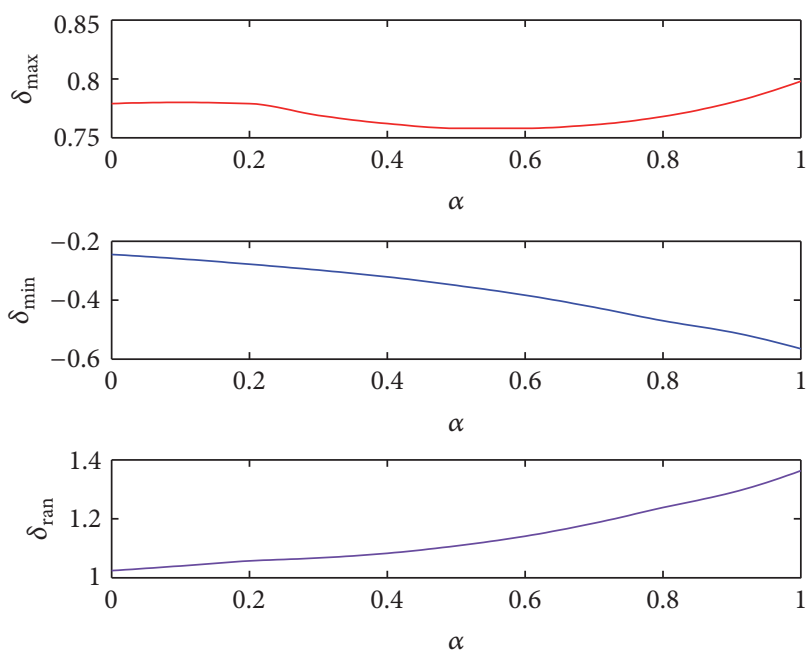

Figure 5: The curves of $\delta_{\max }, \delta_{\min }$, and $\delta_{\text {ran }}$ along with $\alpha$.

TABLE 4: The allowable range $\delta_{\text {ran }}$ for different pair $\left(\epsilon_{1}, \epsilon_{2}\right)$.

\begin{tabular}{ccccc}
\hline & $\epsilon_{2}=0.1$ & $\epsilon_{2}=0.4$ & $\epsilon_{2}=0.6$ & $\epsilon_{2}=0.9$ \\
\hline$\epsilon_{1}=0.1$ & 1.2300 & 1.2300 & 1.2300 & 1.3050 \\
$\epsilon_{1}=0.4$ & 1.1410 & 1.1410 & 1.1410 & 1.1410 \\
$\epsilon_{1}=0.6$ & 1.0900 & 1.0890 & 1.0890 & 1.0890 \\
$\epsilon_{1}=0.9$ & 1.0430 & 1.0430 & 1.0430 & 1.0430 \\
\hline
\end{tabular}

TABLE 5: The allowable range $\delta_{\text {ran }}$ for different pair $\left(\epsilon_{d 1}, \epsilon_{d 2}\right)$.

\begin{tabular}{ccccc}
\hline & $\epsilon_{d 2}=0.1$ & $\epsilon_{d 2}=0.4$ & $\epsilon_{d 2}=0.6$ & $\epsilon_{d 2}=0.9$ \\
\hline$\epsilon_{d 1}=0.1$ & 1.1750 & 1.1700 & 1.1740 & 1.1650 \\
$\epsilon_{d 1}=0.4$ & 1.1300 & 1.1290 & 1.1290 & 1.1280 \\
$\epsilon_{d 1}=0.6$ & 1.1110 & 1.1110 & 1.1110 & 1.1110 \\
$\epsilon_{d 1}=0.9$ & 1.0880 & 1.0880 & 1.0880 & 1.0870 \\
\hline
\end{tabular}

Finally, in order to further demonstrate the utility and superiority of the proposed partially delayed saturated controller (9), some additional comparisons will be done. Based on the above results, it is seen that larger $\alpha$ could lead to less conservative results. In other words, time delay $d$ in controller (9) has a negative effect. In this sense, it is said that the traditionally saturated controller without delay designed for delay systems such as $[35,43]$ will be better in terms of less conservatism. However, it is assumed that system state $x(k)$ of such controllers should be available online. It will be impossible or will be of high cost to satisfy this assumption. On the contrary, saturated controller (9) proposed here can be realized without this assumption. In this sense, it is said that the established method has a larger application scope. On the other hand, it should be pointed out that for a delay system it is not a deterministic conclusion that a saturated controller without delay is better than one with delay. Without loss of generality, when matrix $A_{2}$ is selected to be

$$
A_{2}=\left[\begin{array}{cc}
0.2 & 0.462 \\
0.1 & -0.3
\end{array}\right]
$$




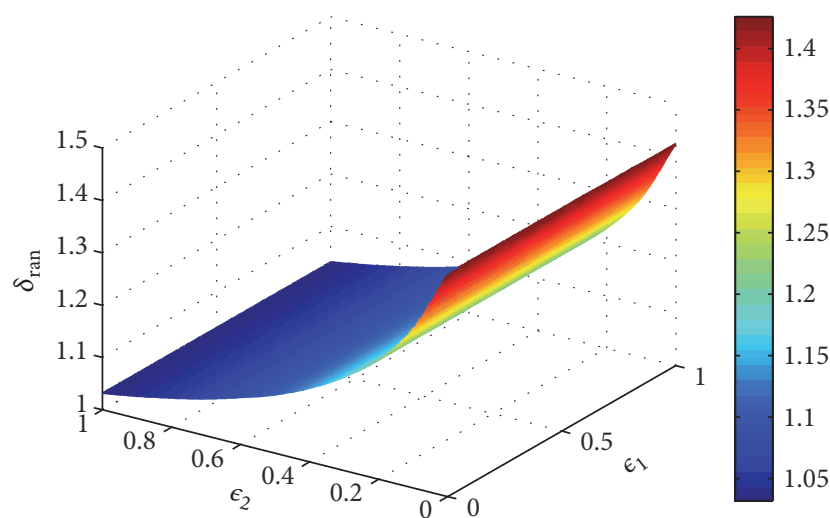

Figure 6: The simulation of correlation between pair $\left(\epsilon_{1}, \epsilon_{2}\right)$ and $\delta_{\text {ran }}$.

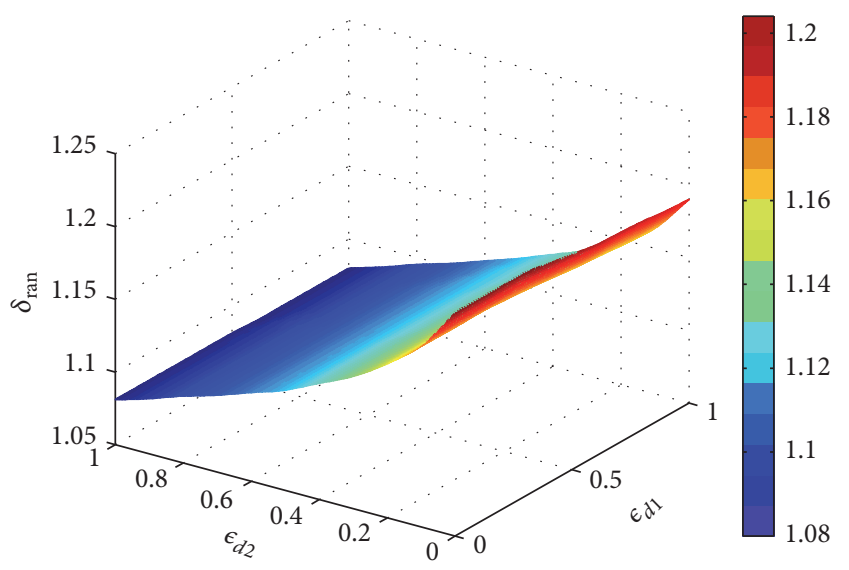

FIGURE 7: The simulation of correlation between pair $\left(\epsilon_{d 1}, \epsilon_{d 2}\right)$ and $\delta_{\text {ran }}$.

and the other parameters with $\delta=0$ are invariant, by Theorem 7 , it is concluded that there is a solution to partially delayed saturated controller (9) only if $\alpha \in[0,0.364]$. For this case, it is seen that larger $\alpha$ will lead to more conservative results in terms of no solvable solution. Particularly, when $\alpha=1$, partially delayed saturated controller (9) will be reduced to be a traditionally saturated controller which could be computed by [44] and is more conservative. However, from this example with different $A_{2}$, it is said that time delay will have a positive effect, which will result in less conservative results. All the comparisons directly show the necessity and superiority of the proposed partially delayed saturated controller.

\section{Conclusion}

In this paper, we have studied the finite-time stabilization problem of discrete-time delayed Markovian jump systems, where the desired saturated controller is partially delaydependent. Firstly, a finite-time stability condition is presented by exploiting a mode-dependent parameter approach. Then, sufficient existence conditions for the partially delaydependent saturated controller are presented with LMIs, which could be solved easily and directly. The correlations between time delay and mode-dependent parameters are considered in detail. Finally, a numerical example is used to demonstrate the effectiveness and advantages of the proposed methods.

\section{Competing Interests}

The authors declare that there are no competing interests regarding the publication of this paper.

\section{Acknowledgments}

This work was supported by the National Natural Science Foundation of China under Grants 61104066, 61374043, and 61473140, the China Postdoctoral Science Foundation funded project under Grant 2012M521086, the Program for Liaoning Excellent Talents in University under Grant LJQ2013040, and the Natural Science Foundation of Liaoning Province under Grant 2014020106.

\section{References}

[1] A. Tejada, O. R. Gonzalez, and W. S. Gray, "Stability analysis of stochastic hybrid jump linear systems using a Markov kernel approach," IEEE Transactions on Automatic Control, vol. 58, no. 12, pp. 3156-3168, 2013.

[2] M. S. Mahmoud and A. A. Saif, "Dissipativity analysis and design for uncertain Markovian jump systems with timevarying delays," Applied Mathematics and Computation, vol. 219, no. 18, pp. 9681-9695, 2013.

[3] J. W. Xia, C. Y. Sun, X. Teng, and H. B. Zhang, "Delay-segmentdependent robust stability for uncertain discrete stochastic Markovian jumping systems with interval time delay," International Journal of Systems Science, vol. 45, no. 3, pp. 271-282, 2014.

[4] G. L. Wang, Q. L. Zhang, and X. G. Yan, Analysis and Design of Singular Markovian Jump Systems, Springer, Basel, Switzerland, 2015.

[5] G. L. Wang, S. Y. Xu, and Y. Zou, "Stabilisation of hybrid stochastic systems by disordered controllers," IET Control Theory \& Applications, vol. 8, no. 13, pp. 1154-1162, 2014.

[6] Y.-Q. Wu, H. Y. Su, R. Q. Lu, Z.-G. Wu, and Z. Shu, "Passivitybased non-fragile control for Markovian jump systems with aperiodic sampling," Systems and Control Letters, vol. 84, pp. 3543, 2015.

[7] G. L. Wang, Q. L. Zhang, and C. Y. Yang, "Stabilization of singular Markovian jump systems with time-varying switchings," Information Sciences, vol. 297, pp. 254-270, 2015.

[8] Y. L. Wei, J. B. Qiu, and M. Wang, "New approach to delaydependent $H_{\infty}$ filtering for discrete-time Markovian jump systems with time-varying delay and incomplete transition descriptions," IET Control Theory and Applications, vol. 7, no. 5, pp. 684-696, 2013.

[9] Z. G. Wu, P. Shi, H. Y. Su, and J. Chu, "Asynchronous $L_{2}-L_{\infty}$ filtering for discretetime stochastic Markov jump systems with randomly occurred sensor nonlinearities," Automatica, vol. 50, no. 1, pp. 180-186, 2014.

[10] G. L. Wang, P. Zhang, and Q. L. Zhang, "A generalized robust $H_{\infty}$ filtering for singular Markovian jump systems and its 
applications," International Journal of Robust and Nonlinear Control, vol. 24, no. 18, pp. 3491-3507, 2014.

[11] H. Shen, Z.-G. Wu, and J. H. Park, "Reliable mixed passive and $H_{\infty}$ filtering for semi-Markov jump systems with randomly occurring uncertainties and sensor failures," International Journal of Robust and Nonlinear Control, vol. 25, no. 17, pp. 32313251, 2015.

[12] L. G. Wu, X. J. Su, and P. Shi, "Output feedback control of Markovian jump repeated scalar nonlinear systems," IEEE Transactions on Automatic Control, vol. 59, no. 1, pp. 199-204, 2014.

[13] L. Liu, X.-D. Zhao, B. Niu, H.-Q. Wang, and X.-J. Xie, "Global output-feedback stabilisation of switched stochastic non-linear time-delay systems under arbitrary switchings," IET Control Theory \& Applications, vol. 9, no. 2, pp. 283-292, 2015.

[14] H. Y. Li, P. Shi, D. Y. Yao, and L. G. Wu, "Observer-based adaptive sliding mode control for nonlinear Markovian jump systems," Automatica, vol. 64, pp. 133-142, 2016.

[15] H. Shen, Y. Zhu, L. Zhang, and J. H. Park, "Extended dissipative state estimation for markov jump neural networks with unreliable links," IEEE Transactions on Neural Networks and Learning Systems, 2016.

[16] G. L. Wang and Q. L. Zhang, "Adaptive state estimation for stochastic delay systems with state-dependent Markovian switching," IET Control Theory \& Applications, vol. 6, no. 6, pp. 822-828, 2012.

[17] H. Y. Li, H. J. Gao, P. Shi, and X. D. Zhao, "Fault-tolerant control of Markovian jump stochastic systems via the augmented sliding mode observer approach," Automatica, vol. 50, no. 7, pp. 1825-1834, 2014.

[18] Z.-G. Wu, P. Shi, H. Y. Su, and J. Chu, "Stochastic synchronization of Markovian jump neural networks with time-varying delay using sampled data," IEEE Transactions on Cybernetics, vol. 43, no. 6, pp. 1796-1806, 2013.

[19] H. Shen, J. H. Park, Z.-G. Wu, and Z. Zhang, "Finite-time $H_{\infty}$ synchronization for complex networks with semi-Markov jump topology," Communications in Nonlinear Science and Numerical Simulation, vol. 24, no. 1-3, pp. 40-51, 2015.

[20] G. P. Chen, Y. Yang, and Q. N. Pan, "Finite time stability analysis of switched systems with stable and unstable subsystems," Asian Journal of Control, vol. 16, no. 4, pp. 1224-1228, 2014.

[21] Z. Zhang, Z. X. Zhang, H. Zhang, B. Zheng, and H. R. Karimi, "Finite-time stability analysis and stabilization for linear discrete-time system with time-varying delay," Journal of the Franklin Institute, vol. 351, no. 6, pp. 3457-3476, 2014.

[22] X. Xing, D. Y. Yao, Q. Lu, and X. C. Li, "Finite-time stability of Markovian jump neural networks with partly unknown transition probabilities," Neurocomputing, vol. 159, no. 1, pp. 282-287, 2015.

[23] Z. G. Yan, W. H. Zhang, and G. S. Zhang, "Finite-time stability and stabilization of Itô stochastic systems with Markovian switching: mode-dependent parameter approach," IEEE Transactions on Automatic Control, vol. 60, no. 9, pp. 2428-2433, 2015.

[24] Z. Yan, G. Zhang, and W. Zhang, "Finite-time stability and stabilization of linear Itô stochastic systems with state and control-dependent noise," Asian Journal of Control, vol. 15, no. 1, pp. 270-281, 2013.

[25] J. Fu, R. C. Ma, and T. Y. Chai, "Global finite-time stabilization of a class of switched nonlinear systems with the powers of positive odd rational numbers," Automatica, vol. 54, pp. 360-373, 2015.
[26] S. P. He and F. Liu, "Finite-time $H_{\infty}$ fuzzy control of nonlinear jump systems with time delays via dynamic observer-based state feedback," IEEE Transactions on Fuzzy Systems, vol. 20, no. 4, pp. 605-614, 2012.

[27] Y. Q. Zhang, C. X. Liu, and X. W. Mu, "Robust finite-time $H_{\infty}$ control of singular stochastic systems via static output feedback," Applied Mathematics and Computation, vol. 218, no. 9, pp. 5629-5640, 2012.

[28] Y. Ma and H. Chen, "Reliable finite-time $H_{\infty}$ filtering for discrete time-delay systems with Markovian jump and randomly occurring nonlinearities," Applied Mathematics and Computation, vol. 268, pp. 897-915, 2015.

[29] F. Amato, G. Carannante, G. D. Tommasi, and A. Pironti, "Input-output finite-time stability of linear systems: necessary and sufficient conditions," IEEE Transactions on Automatic Control, vol. 57, no. 12, pp. 3051-3063, 2012.

[30] F. Amato, G. De Tommasi, and A. Pironti, "Input-output finitetime stabilization of impulsive linear systems: necessary and sufficient conditions," Nonlinear Analysis: Hybrid Systems, vol. 19, pp. 93-106, 2016.

[31] T. Hu, Z. Lin, and B. M. Chen, "Analysis and design for discretetime linear systems subject to actuator saturation," Systems \& Control Letters, vol. 45, no. 2, pp. 97-112, 2002.

[32] J. M. G. da Silva and S. Tarbouriech, "Anti-windup design with guaranteed regions of stability for discrete-time linear systems," Systems \& Control Letters, vol. 55, no. 3, pp. 184-192, 2006.

[33] T. Alamo, A. Cepeda, D. Limon, and E. F. Camacho, "Estimation of the domain of attraction for saturated discrete-time systems," International Journal of Systems Science, vol. 37, no. 8, pp. 575583, 2006.

[34] Z. L. Lin and L. Lv, "Set invariance conditions for singular linear systems subject to actuator saturation," IEEE Transactions on Automatic Control, vol. 52, no. 12, pp. 2351-2355, 2007.

[35] L. X. Zhang, E.-K. Boukas, and A. Haidar, "Delay-rangedependent control synthesis for time-delay systems with actuator saturation," Automatica, vol. 44, no. 10, pp. 2691-2695, 2008.

[36] X. Ji, Y. Sun, and H. Su, "Analysis and design for singular discrete linear systems subject to actuator saturation," Asian Journal of Control, vol. 13, no. 2, pp. 350-355, 2011.

[37] H. P. Liu, E. K. Boukas, F. C. Sun, and D. W. C. Ho, "Controller design for Markov jumping systems subject to actuator saturation," Automatica, vol. 42, no. 3, pp. 459-465, 2006.

[38] S. P. Ma, C. H. Zhang, and S. Q. Zhu, "Robust stability for discrete-time uncertain singular Markov jump systems with actuator saturation," IET Control Theory \& Applications, vol. 5, no. 2, pp. 255-262, 2011.

[39] S. P. Ma and C. H. Zhang, " $H_{\infty}$ control for discrete-time singular Markov jump systems subject to actuator saturation," Journal of the Franklin Institute, vol. 349, no. 3, pp. 1011-1029, 2012.

[40] T. Shi, H. Su, and J. Chu, "Robust $H_{\infty}$ output feedback control for Markovian jump systems with actuator saturation," Optimal Control Applications \& Methods, vol. 33, no. 6, pp. 676-695, 2012.

[41] C. A. C. Gonzaga and O. L. V. Costa, "Stochastic stabilization and induced $\ell_{2}$-gain for discrete-time Markov jump Lur'e systems with control saturation," Automatica, vol. 50, no. 9, pp. 2397-2404, 2014.

[42] J. M. G. da Silva and S. Tarbouriech, "Antiwindup design with guaranteed regions of stability: an LMI-based approach," IEEE Transactions on Automatic Control, vol. 50, no. 1, pp. 106-111, 2005. 
[43] B. Y. Zhang, S. Y. Xu, Y. S. Zhang, and Y. M. Chu, "Delaydependent stabilization for delayed Markovian jump systems subject to input saturation," in Proceedings of the 29th Chinese Control Conference (CCC '10), pp. 5981-5986, Beijing, China, July 2010.

[44] L. Sun, Y. Wang, and G. Feng, "Control design for a class of affine nonlinear descriptor systems with actuator saturation," IEEE Transactions on Automatic Control, vol. 60, no. 8, pp. 21952200, 2015.

[45] Z. Zhang, W. Liu, and D. Zhou, "Global asymptotic stability to a generalized Cohen-Grossberg BAM neural networks of neutral type delays," Neural Networks, vol. 25, pp. 94-105, 2012.

[46] Z. Q. Zhang, J. D. Cao, and D. M. Zhou, "Novel LMI-based condition on global asymptotic stability for a cass of CohenGrossberg BAM networks with extended activation functions," IEEE Transactions on Neural Networks and Learning Systems, vol. 25, no. 6, pp. 1161-1172, 2014. 


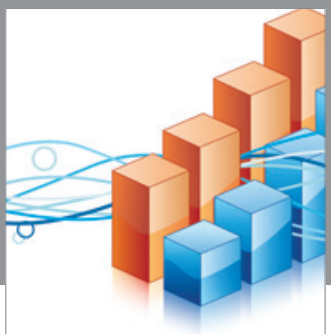

Advances in

Operations Research

vatem alat4

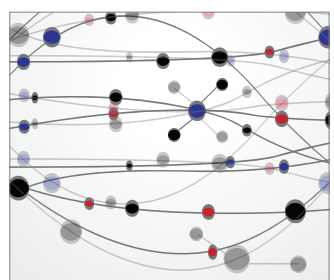

\section{The Scientific} World Journal
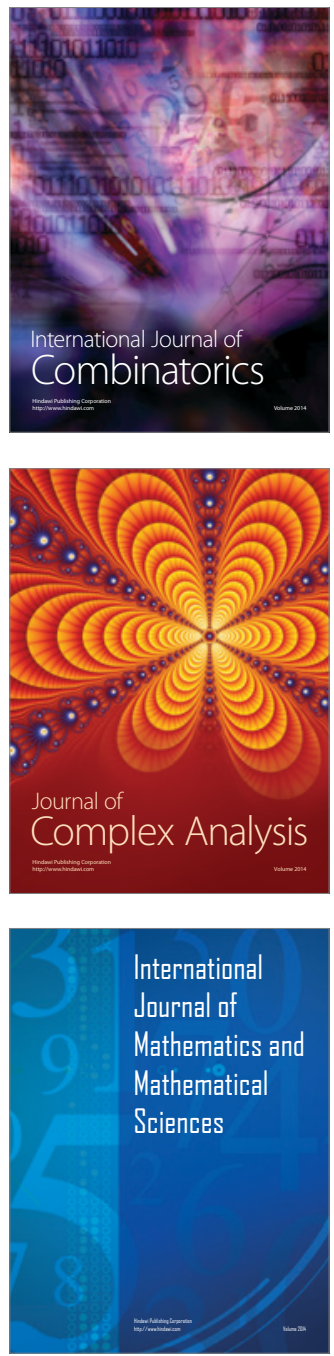
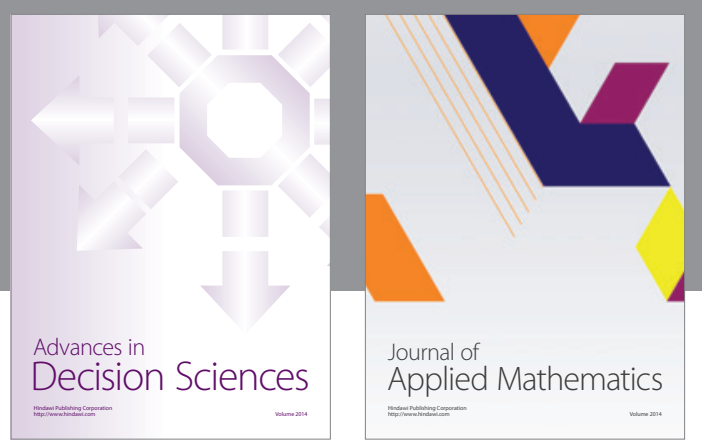

Algebra

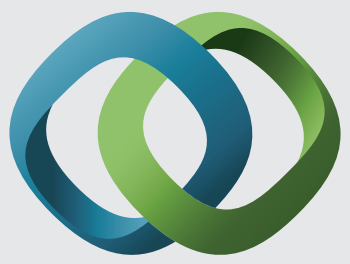

\section{Hindawi}

Submit your manuscripts at

http://www.hindawi.com
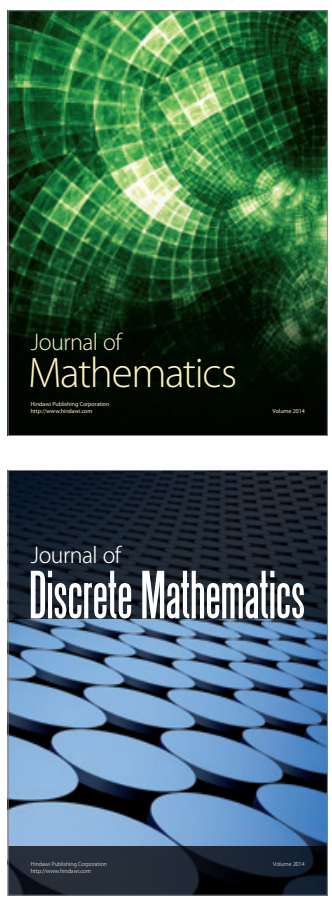

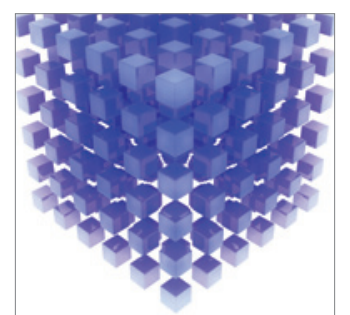

Mathematical Problems in Engineering
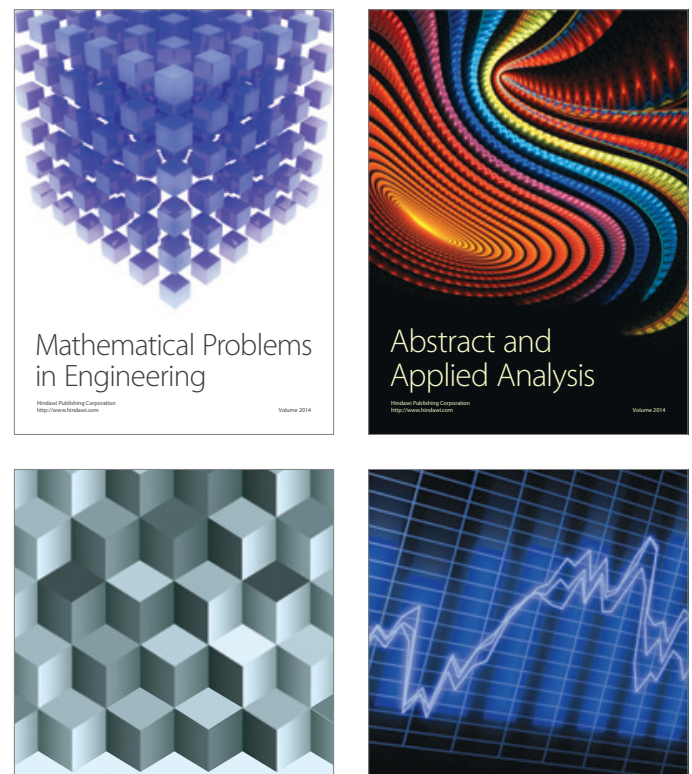

Journal of

Function Spaces

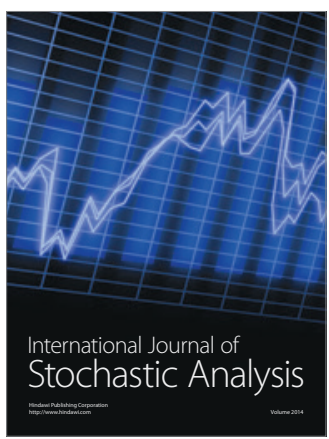

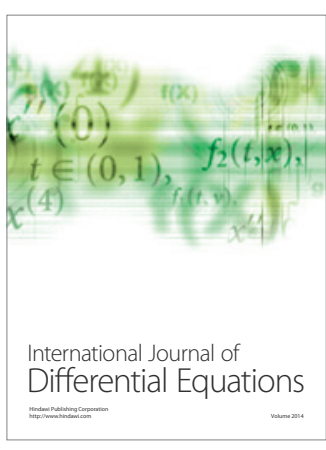
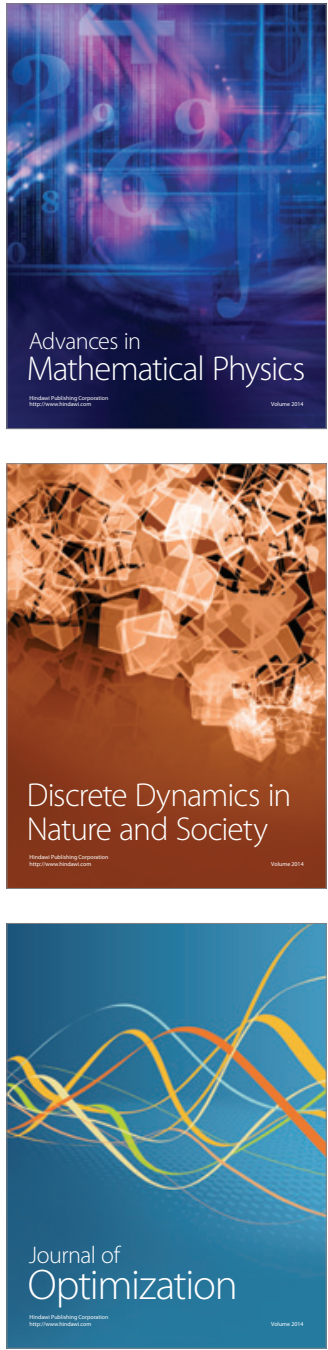